\title{
Troponin-I release after cardiac surgery with different surgical techniques and post-operative neurological outcomes
}

\author{
Norzeihan Jan Bappu*, Panangipalli Venugopal, Akhshay Kumar Bisoi, Pankaj S \\ Mankad
}

\begin{abstract}
Cerebral hypoperfusion during cardiopulmonary bypass surgery has been thought to be a factor in the aetiology of brain damage with evidence of post-operative neurological deficits. Cardiacspecific biomarkers such as troponin-I, troponin-T and CK-MB have been used extensively to predict myocardial injury and ischaemia. This prospective study investigateed the level of troponin-I release in both off-pump and CPB-technique CABG surgery, as well as postulated a relationship of troponin release and post-operative neurological outcome. A total of 44 adult patients undergoing coronary artery bypass graft (CABG) were enrolled into either an off-pump or on-pump groups, with 22 patients participating in each. Group A (on-pump) underwent myocardial revascularisation with CPB and cardioplegic arrest, while Group B (off pump) underwent beating heart surgery. The measurement of troponin-I is a 1-step enzyme immunoassay method, with specificity and sensitivity set at $0.4 \mathrm{ug} / \mathrm{mL}$. Neurological assessment was done using the NIH Stroke Scale, and neuropsychologic assessment was assessed on cognitive function using modified Weschler Memory Scale, for which scores were standardized to achieve a composite measure of concentration. A set of statistical analysis was done to correlate troponin-I release with different surgical techniques of CPB and OPCAB. Although each independent technique showed a marked rise of troponin-I from baseline to 6 hours post-operatively, the difference in troponin release was not significant between the 2 groups at specified time intervals $(p=0.124)$. There was however a significant correlation of troponin-I release with the number of grafts used in the surgery, irrespective of the type of grafts or surgical technique. None of the patients in either group showed any neurological or cognitive deficits presenting at day 3 and day 7 post-operatively. The findings of this study demonstrate that there is no significant short-term cognitive or neurological dysfunctions post-operatively, as indicated by troponin-I release in assessing the severity of myocardial injury.
\end{abstract}

\section{INTRODUCTION}

Cerebral damage associated with cardiac surgery has been extensively studied and this has been

* To whom correspondence should be addressed: Norzeihan Jan Bappu. Department of Cardiothoracic Surgery, The Royal Infirmary of Edinburgh, Little France, Crescent, Old Dalkeith Road, Edinburgh, Scotland, United Kingdom, EH16 4SU

Email: jan.bappu@luht.scot.nhs.uk comprehensively reviewed (1-3). Cerebral hypoperfusion during cardiopulmonary bypass surgery has long been thought to be a factor in the aetiology of brain damage with evidence of post-operative neurological deficits $(2,3)$. Cerebral injury may also occur in the early post-operative period, or alternatively, any intraoperative damage may be exacerbated by hypoperfusion at this stage. 
Previous studies have shown that cardiac surgery using extracorporeal circuit, also known as the cardiopulmonary bypass (CPB) technique, is associated with higher intraoperative myocardial damage and hence consecutively, worse cerebral hypoperfusion (46 ). There is a general consensus recently to undertake off-pump or beating heart coronary artery bypass graft (CABG) surgery with prospective aim that by avoiding $\mathrm{CPB}$, cerebral and other complications should be limited. However, the incidence of cerebral complications after cardiac surgery varies considerably depending on the patients' demographical characteristics such as age, timing of the post-operative assessment, sensitivity of the assessment procedures and whether prospective or retrospective study procedures were used (7).

Current research showed that apart from a higher incidence of cerebral hypoperfusion and ischaemia, CPB produces numerous important causes of myocardial damage such as atriotomy, poor myocardial protection, duration of aortic cross-clamping, myocardial stunning; as well as myocardial ischaemia and infarction (7-9). During cardiac operations with $\mathrm{CPB}$, the heart is arrested and protected using cardioplegia; during this period the heart is rendered ischaemic. At the end of CPB, the heart is reperfused and the cardiac action resumes. This reperfusion after ischaemic period produces myocardial damage and eventual necrosis. By contrast, during an off-pump cardiac surgery, the heart keeps beating and thus reperfusion injury is avoided.

Cardiac-specific biomarkers such as troponin-I, troponin-T and CK-MB, have been used extensively until recently, to predict myocardial injury and ischaemia (10-15). Troponin-I is solely confined to the myocardium and has been shown to be a highly specific marker for the detection of myocardial injury.

The aim of this prospective study was to investigate the level of troponin-I release in both off-pump and CPB-technique CABG surgery, and determine whether there is a significant myocardial injury associated with $\mathrm{CPB}$, as suggested by the literature. This study also scrutinised the relationship of troponin-I with postoperative neurological sequelae in both $\mathrm{CPB}$ and OPCAB groups.

\section{METHODS}

This study was carried out in Department of Cardiothoracic Surgery, All India Institute of Medical Sciences, with subsequent data revision done in the New Royal Infirmary of Edinburgh. The study was approved by the Indian Medical Research, New Delhi.

A total of 44 adult patients undergoing coronary artery bypass graft (CABG) were enrolled into either an off-pump or on-pump groups, with 22 patients participating in each. Group A (on-pump) underwent myocardial revascularisation with $\mathrm{CPB}$ and cardioplegic arrest, while Group B (off pump) underwent beating heart surgery. Age boundaries were set at 16 and 85 years. On preoperative admission, the study was fully discussed with the patient, clerking on history and clinical examination performed. Preoperative documentation included patient's age, gender, patient's pathology, operation planned, anti-platelet therapy given within seven days, pre-operative anticoagulation and bleeding history. The inclusion criteria for patients in the study were; stable angina not suitable for balloon angioplasty or stenting, with two or more vessel disease. Exclusion criteria were the inability to provide informed consent, acute infections, severe ongoing neurological dysfunctions, impaired left ventricular fraction with ejection fraction of less than $30 \%$, recent myocardial infarction within 1 month, acute renal failure, previous stroke or transient ischaemic attack, coagulopathy, emergency/ redo surgery and surgical re-exploration. This study conforms to the Declaration of Helsinki as regards medical research on human subjects as well as local ethical requirements.

\section{Patient management}

Standard monitoring was instituted prior to surgery; arterial line, central venous pressure, ECG, urinary catheter and nasal temperature.

All previous cardiac medications were continued up to the day of surgery, except aspirin and clopidogrel, which were stopped a week before the operation. Anaesthetic agents used were kept standard for all patients in both groups. Intravenous anaesthesia with propofol infusion 3mg.kg.hour and fentanyl 1 $\mathrm{mg} / \mathrm{kg} / \mathrm{min}$ were given at induction. Neuromuscular blockage was achieved by pancuronium bromide 0.15 mg.kg and lung ventilation was maintained as normocapnia with oxygen and air, without positive end expiratory pressure (PEEP).

Intraoperative evaluations were recorded on haemodynamic stabilility of the patients, the type and number of grafts used, the duration of the surgery, and specifically in CPB group, the duration of the CBP and aortic cross-clamp time.

Body surface area was calculated as a variable in patient demographic data, using the Dubois and Dubois Formula:

Body surface area $\left(\mathrm{m}^{2}\right)=0.20247 \mathrm{x}$ height $(\mathrm{m})^{0.725} \mathrm{x}$ weight $(\mathrm{kg})^{0.425}$

\section{Operative technique}

Standard open-heart technique was performed for all patients through total median sternotomy. In patients 
undergoing surgery with $\mathrm{CPB}$, heparin was added to the pump prime at a concentration of $1 \mathrm{iu} / \mathrm{ml}$. Prior to the institution of cardiopulmonary bypass (CPB) heparin was administered to the patient at a dosage of $300 \mathrm{iu} / \mathrm{Kg}$ sufficient to raise the accelerated clotting time (ACT) to above 480 seconds. Whilst on CPB supplemental heparin was administered as required to maintain the ACT above this level. CPB was established with a roller pump, the use of a flexible venous reservoir with vent and cardiotomy suction. Systemic cooling was carried out to 28-32 C. Cold blood cardioplegia was used following application of the aortic cross clamp to achieve cardiac arrest. Once rewarming to $37 \mathrm{C}$ was completed and $\mathrm{CPB}$ discontinued, protamine sulphate was given to reverse systemic heparinisation. Where the ACT remained above 140 seconds supplemental protamine was given. All pump blood remaining, except for that contained within the extra-corporeal circuit, was returned to the patient. After weaning from CPB, mean arterial pressure was maintained above $60 \mathrm{mmHg}$ with appropriate vasoactive drugs.

In group $\mathrm{A}$, the proximal grafts anastomoses were performed during aortic cross-clamp, and distal anastomoses followed subsequently. For off-pump group (Group B), Octopus device stabiliser were used only once during the graft anastomoses, with mean arterial pressure always greater than $65 \mathrm{mmHg}$ throughout the surgery to maintain haemodynamic stability. Heparin was administered at dosage of 1 $\mathrm{mg} / \mathrm{kg}$, protamine sulphate was not given for reversal of heparinisation. The temperature was set to be normothermia at $36 \mathrm{C}$. Intraoperative time was recorded from the beginning of sternotomy until the end of last distal anastomoses, whereas the surgery time was defined from the start of induction until transfer to the intensive care unit.

Thoracic drains were placed in the mediastinum, left and right pleural cavities where required. Post-operative suction was maintained at $20 \mathrm{~cm} / \mathrm{H} 2 \mathrm{O}$. All red cell transfusions were carried out as per local protocol. Crystalloid was given in the intensive care at a rate of $0.5 \mathrm{mls} / \mathrm{Kg}$ with colloid administered where required.

\section{Troponin-I Measurements}

The measurement of troponin-I is a 1-step enzyme immunoassay method, with specificity and sensitivity of $0.4 \mathrm{ug} / \mathrm{mL}$. The specificity with minimum value above $0.4 \mathrm{ug} / \mathrm{ml}$ is necessary to indicate positive value. Serial measurements were taken at specified time from the arterial line. For patients undergoing surgery with $\mathrm{CPB}$, measurements were taken at induction, 20 minutes after the start of $\mathrm{CPB}$, at skin closure, 6 hours and 24 hours post-operatively. In OPCAB group, measurement was taken at induction, after the distal anastomosis of the graft conduits, at skin closure, 6 hours and 24 hours post-operatively. During the surgery, inspection was made on the condition of the aorta; patients who presented with abnormalities such as atherosclerotic plaque were excluded from the study.

\section{Pre-operative and post-operative evaluations}

Post-operative assessment was recorded into 2 components at day 3 post-operatively: 1) general observation on duration of ICU stay, post-operative ECG changes and re-exploration; 2)neurologic and neuropyshologic assessment. Neurological assessment was done using the NIH Stroke Scale, with patient diagnosed with acute neurological dysfunction if the criteria is above 10 (total score of 22). Any abnormal score would indicate cognitive dysfunction postoperatively, provided the baseline score at pre-operative was normal.

A standardized neurologic examination was done using the NIH Stroke Scale which assessed mentation, cranial nerve function, motor power, reflexes, sensation/cerebellar function, and gait, with 14 individual elements graded on a scale from 0 to 3 for a possible total of 42 was performed the day before the surgery. A score of more than 10 on total baseline assessment was identified as indicating preoperative neurologic dysfunction. Neuropsychologic assessment was done on cognitive function of the patient using modified Weschler Memory Scale, for which scores were standardized and averaged to achieve a composite measure of concentration.

\section{RESULTS}

\section{Clinical characteristics}

The patients' demographic data are shown in Table-2. A total of 44 patients were recruited for the study. One patient in CPB group had to be re-explored due to massive bleeding at day 1 post-operatively. None of the other patients in both groups had any serious bleeding through nose, gastrointestinal tract or urine catheter.

\section{Serial troponin-I measurements}

Below is the troponin values at each time interval of induction, intraoperative (20 minutes after start of CPB in $\mathrm{CPB}$ group, after distal anastomosis in OPCAB group), at skin closure, 6 hours and 24 hours postoperatively.

Table 1. Demographic and pre-operative data ( $\mathrm{n}=22$ in each group)

\begin{tabular}{llll}
\hline Variable & OPCAB group & CPB group & p value \\
\hline Age & $59.6 \pm 8.5$ & $59.1 \pm 9.1$ & NS \\
\hline Sex & 13 males $(60 \%)$, & 15 males $(70 \%)$, &
\end{tabular}




\begin{tabular}{|c|c|c|c|c|}
\hline & \multicolumn{2}{|c|}{9 females $(40 \%)$} & 7 females $(30 \%)$ & NS \\
\hline Weight & \multicolumn{2}{|c|}{$67.0 \pm 7.2 \mathrm{~kg}$} & $65.9 \pm 8.2 \mathrm{~kg}$ & NS \\
\hline Height & \multicolumn{2}{|c|}{$163.5 \pm 7.9 \mathrm{~cm}$} & $161.7 \pm 8.0 \mathrm{~cm}$ & NS \\
\hline \multicolumn{2}{|c|}{$\begin{array}{l}\text { Body } \\
\text { surface area }\left(\mathrm{m}^{2}\right)\end{array}$} & $1.57 \pm 0.83$ & $1.60 \pm 0.26$ & NS \\
\hline \multicolumn{2}{|c|}{$\begin{array}{l}\text { Preoperative } \\
\text { ejection fraction }\end{array}$} & \multicolumn{2}{|c|}{$60 \% \pm 0.359 \% \pm 0.1$} & NS \\
\hline \multicolumn{3}{|c|}{$\begin{array}{l}\text { Preoperative } \\
\text { myocardial infarction }\end{array}$} & \multicolumn{2}{|l|}{9} \\
\hline \multicolumn{2}{|c|}{ X-Clamp } & - & \multicolumn{2}{|l|}{$35.1 \pm 10.6$ minutes } \\
\hline \multicolumn{2}{|c|}{ CPB time } & - & \multicolumn{2}{|l|}{$56.6 \pm 17.9$ minutes } \\
\hline \multicolumn{3}{|c|}{ Minimum Temp } & \multicolumn{2}{|l|}{$31.7 \pm 1.5^{\circ} \mathrm{C}$} \\
\hline \multicolumn{3}{|c|}{ Max rewarming Temp } & \multicolumn{2}{|l|}{$37.2 \pm 0.32{ }^{\circ} \mathrm{C}$} \\
\hline \multicolumn{2}{|c|}{ Prothrombin time } & 1 & 1 & NS \\
\hline
\end{tabular}

Table 2. Intraoperative and Post-operative Data

\begin{tabular}{lll}
\hline Variables & OPCAB & CPB \\
\hline LIMA graft & 22 & 22 \\
\hline Saphenous vein grafts & 20 & 21 \\
\hline $\begin{array}{l}\text { Grafts anastomoses } \\
\text { per patient }\end{array}$ & $3.2 \pm 0.5$ & $3.8 \pm 0.1$ \\
\hline $\begin{array}{l}\text { Ventilation time (min) } \\
\text { Post-operative } \\
\text { ejection fraction ( \% ) }\end{array}$ & $38.4 \pm 13$ & $45.7 \pm 20$ \\
\hline $\begin{array}{l}\text { Sternal infection } \\
\text { Leg wound infection }\end{array}$ & $52 \% \pm 0.2$ & $56 \% \pm 0.3$ \\
\hline $\begin{array}{l}\text { Length of ICU stay (days) } \\
\text { hospital stay (days) }\end{array}$ & $1.5 \pm 0.8$ & 1 \\
\hline \begin{tabular}{l}
-day mortality \\
\hline
\end{tabular} & $2.6 \pm 2.0$ & $1.6 \pm 0.5$ \\
\hline
\end{tabular}

Table 3. Troponin-I values for both groups

\begin{tabular}{lll}
\hline Time Interval & OPCAB $(\mathbf{u g} / \mathbf{m l})$ & CPB $(\mathbf{u g} / \mathbf{m l})$ \\
\hline Induction & $0 \pm 0$ & $0 \pm 0$ \\
\hline Intra-operative & $0.65 \pm 0.85$ & $1.35 \pm 0.75$ \\
\hline Skin closure & $2.05 \pm 1.57$ & $2.35 \pm 1.16$ \\
\hline 6 hours & $2.10 \pm 1.56$ & $5.20 \pm 6.10$ \\
\hline
\end{tabular}

24 hours

$1.95 \pm 1.69$

$4.55 \pm 5.67$

Another set of analysis was made to see the correlation of troponin-I with different surgical techniques of CPB and OPCAB. Surprisingly, although each independent technique showed a marked rise of troponin-I from baseline to 6 hours post-operatively, the troponin release was not significant between the 2 groups at specified time intervals $(\mathrm{p}=0.124)$. Hence, when troponin-I is used as a cardiac biomarker for myocardial injury, troponin release in both groups do not reflect whether one technique is superior to the other based on short term cardiac clinical outcomes. In CPB group, there was no significance between post-operative troponin release with the duration of CPB or the aortic cross-clamp time ( $\mathrm{p}>0.05)$.

Troponin level rise was almost uniform in both groups. This exception was noted in one patient in CPB group, where her post-operative 6 hours level was $22 \mathrm{ug} / \mathrm{ml}$. This patient had severe syncope preceded by palpitation 2 weeks prior to surgery, diagnosed as sick

Fig-1:Troponin-I Level at Specified Time Intervals

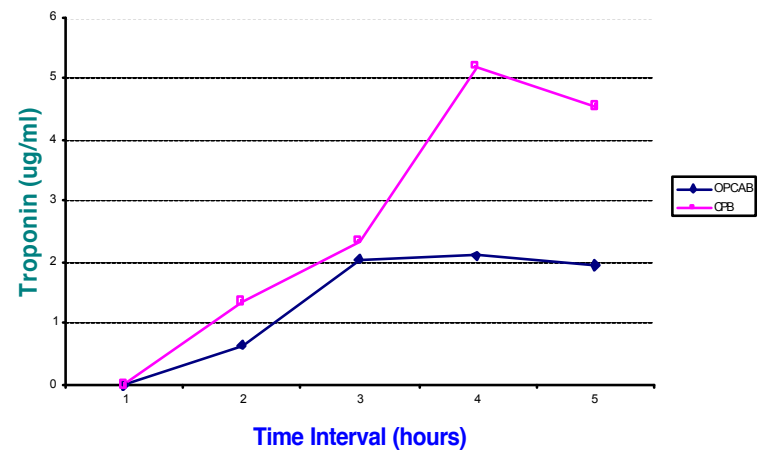

sinus syndrome and was put on temporary pacemaker up to the day of operation. The patient had bilateral renal artery stenosis and 2 episodes of myocardial infarction within a year prior to surgery. Although her baseline troponin-I was undetected, it was concluded that her co-morbidity prior to surgery contributed to significant myocardial injury intra-operatively.

\section{Number of grafts and troponin-I release}

There was a significant correlation of troponin-I release with the number of grafts used in the surgery, irrespective of the type of neither the grafts nor the surgical technique. Graph below shows the statistical correlation between the number of grafts and the troponin release at 6 hours post-operatively. (Figure 2; $60 \%$ Pearson Correlation with $\mathrm{p}=0.005$ ).

\section{Post-operative evaluations}

On general observation, there was a longer ICU stay 
in patients with higher troponin release at 6 hours after closure (Pearson Correlation 79.2\%, $\mathrm{p}<0.05$ ). However, upon specific observations, it was noted that the length of ICU stay was not entirely determined by patients' haemodynamic stability, but also dependent on bed availability and date of transfer to the ward. Therefore, other factors should be kept into considerations before troponin is used as a sole predictor to determine patients' haemodynamic stability and length of ICU stay.

Patients in both groups underwent thorough neurologic and neuropsychologic clinical assessment at day 3 and day 7 after surgery. None of the patients in both groups showed any neurological deficits or cognitive deficits presenting post-operatively. This information indicates that any adverse effects on myocardial injury are normalised and were not followed

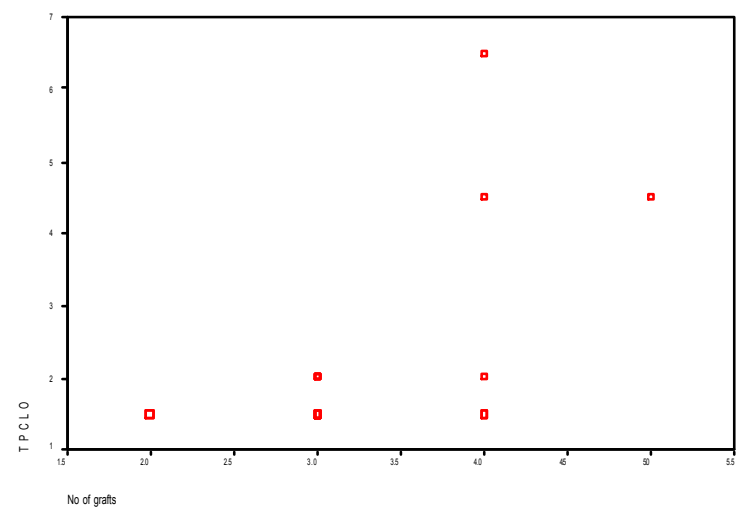

by any acute sequelae, representing deficit of operative procedures. With this negative finding on acute neurological outcomes, there was no significant relationship on biochemistry evaluation of troponin-I with acute post-operative neurological outcomes. Long term neurological outcome was not however being carried out for the purpose of this study.

\section{DISCUSSION}

Although the current consensus is with renewed interest and in favour of OPCAB technique in cardiac surgery, there has always been a debate on which surgical technique is more superior to another, in reducing the morbidity and mortality owing to myocardial revascularisation. One definite advantage of OPCAB is with increasing surgical demand; coronary operations could be performed at a lower cost without the expenses of a cardio-pulmonary bypass machine. However, a major concern on undertaking an off-pump approach is the obvious difficulty of performing the coronary anastomoses on the beating heart, with an increased risk of intraoperative myocardial ischaemia and suboptimal anastomoses performed (17). It goes without saying that achieving complete revascularisation should be the main priority of the surgery irrespective of the technique, as incomplete anastomoses of the grafts is significantly associated with post-operative mortality rate, perioperative myocardial infarction, and low cardiac output $(17,18)$. It is also important to consider other factors when choosing surgical procedure to be performed; this include surgeon's degree of expertise, patient population and their pre-existing comorbidities.

Biochemical markers have been used extensively to identify the mechanism of myocardial injury associated with surgical technique and the post-operative neurological sequelae, for patients who have had cardiac surgery performed with or without CPB. Recent literature suggests the superiority of OPCAB technique versus $\mathrm{CPB}$, with relations to reducing morbidity and mortality associated with myocardial injury and cerebral hypoperfusion associated with the use of extracorporeal circuit of CPB $(4,5)$. Hypoperfusion during $\mathrm{CPB}$ is hypothesised to be related to microembolism and inflammatory changes that lead to increase in blood-brain barrier permeability, resulting in cerebral oedema. Hence, this study was carried out to confirm the previous results and to determine if there are any acute post-operative neurological deficits in CPB group.

The finding of this study does not support the current consensus, as we found that although there is a definite rise of troponin-I in both groups 6 hours after sternal closure, there is no significant difference in troponin release between $\mathrm{CPB}$ and $\mathrm{OPCAB}$ groups. It is important to note that there are multiple factors involved in contributing to inflammatory injury, apart from CPB, such as surgical trauma, ischaemicreperfusion injury and thrombin activation (19). Surgery alone may activate haemostatic responses, activation of immune mechanisms and inflammatory responses mediated by the release of various cytokines and chemokines. Hence as seen in this study, the rise in troponin was almost uniform post-operatively in both group, with definite evidence of myocardial injury as the result of surgery itself.

With respect to inflammatory response, it is difficult to draw a conclusion on the extent of myocardial injury based on biochemical parameters alone, as there are other several confounding factors that may interplay in determining the ultimate haemostatic derangement. The threshold for inflammatory mediator release is also determined by patient individual risk stratification, protocol of heparin and protamine administration, anaesthesiologic techniques, and the expertise of the surgeon operating the cases. Hence troponin rise in this study does not indicate a direct correlation between the inflammatory response and the actual physiologic 
mechanisms.

The study by Ascione et al suggests that complement activation of IL-6 is similar in both off-pump and onpump patients after the cardiac surgery (20). This evidence is important to support the concept that surgical injury per se triggers release of acute-phase reactants, with possible impact on clinical consequences.

On a positive note, the statistical analysis however shows that irrespective of the surgical procedure, there is a significant positive correlation between the number of grafts performed in the surgery with 6-hour postoperative troponin-I release. (60\% Pearson Correlation with $\mathrm{p}=0.005$ ). Considering that the duration of the surgery increases on linear pattern with the number of grafts for each case, it is not surprising that myocardial injury is significantly higher in relation to the actual operation time, and the extent of anastomoses done directly on the myocardium. There is no significant difference between the troponin release and the type of grafts performed, such as LIMA, saphenous vein artery or radial grafts.

The evaluation of postoperative morbidity shows positive correlation between the rise of troponin after 6 hours post-operatively and the length of ICU stays in both groups of patients. Despite the optimism to use troponin as a biomarker for acute post-operative neurological deficit, the population of the study did not show any acute, evolving neurological symptoms as the result of the surgery. More follow-ups are needed to investigate whether these patients may show significant clinical signs on long term outcome. It should be emphasised that apart from clinical evaluation, there is a lack of effective methodology or marker for assessing neurologic and cognitive dysfunctions after cardiac surgery.

At present, more analysis is needed to explore the surgical complications of these patients, in terms of graft patency for CPB versus OPCAB groups, and also long term prospective neurological outcome after CPBtechnique, as suggested by previous studies. Major postoperative complications such as in-hospital mortality was rare, hence large cohort studies would be needed to identify those aspects between the two groups in order to achieve positive statistical differences.

The findings of this study demonstrate that there is no proven significant short term cognitive or acute neurological dysfunctions post-operatively, as indicated by troponin-I in assessing the severity of myocardial injury, within the scope of our research. Although this contradicts the popular belief that off-pump surgery reduces morbidity and mortality rate, these observations may encourage a larger cohort in the future to improve our understanding on the actual mechanism of myocardial injury, without omitting the judicious stratification of the patient's pre-existing clinical condition in determining the most appropriate surgical technique for their own optimal outcome.

\section{REFERENCES}

1. Kuroda Y, Uchimoto R, Kaieda R, Shinkura R, Shinohara K, Miyamoto S, Oshita S, Takeshita H. Central nervous system complications after cardiac surgery: a comparison between coronary artery bypass grafting and valve surgery. Anesthesia \& Analgesia. 1993; 76:222-227.

2. Millar SM, Alston RP, Andrews PJ, Souter MJ. Cerebral hypoperfusion in immediate postoperative period following coronary artery bypass grafting, heart valve, and abdominal aortic surgery. British Journal of Anaesthesia. 2001; 87:229-236.

3. Fearn SJ, Pole R, Wesnes K, Faragher EB, Hooper TL, McCollum CN. Cerebral injury during cardiopulmonary bypass: emboli impair memory. Journal of Thoracic \& Cardiovascular Surgery. 2001; 121:1150-1160.

4. Schneider M, Valentine S, Hegde RM, Peacock J, March S, Dobb GJ. The effect of different bypass flow rates and low-dose dopamine on gut mucosal perfusion and outcome in cardiac surgical patients.[erratum appears in Anaesth Intensive Care 1999 Apr;27(2):228]. Anaesthesia \& Intensive Care. 1999; 27:13-19.

5. Taylor KM. Central nervous system effects of cardiopulmonary bypass. Annals of Thoracic Surgery. 1998; 66:Suppl-4.

6. Stump DA, Rogers AT, Hammon JW, Newman SP. Cerebral emboli and cognitive outcome after cardiac surgery. Journal of Cardiothoracic \& Vascular Anesthesia. 1996; 10:113-118.

7. Furlan AJ, Sila CA, Chimowitz MI, Jones SC. Neurologic complications related to cardiac surgery. Neurologic Clinics. 1992; 10:145-166.

8. Utley JR. Techniques for avoiding neurologic injury during adult cardiac surgery. Journal of Cardiothoracic \& Vascular Anesthesia. 1996; 10:38-43.

9. Kuroda Y, Uchimoto R, Kaieda R, Shinkura R, Shinohara K, Miyamoto S, Oshita S, Takeshita H. Central nervous system complications after cardiac surgery: a comparison between coronary artery bypass grafting and valve surgery. Anesthesia \& Analgesia. 1993; 76:222-227.

10. Nagurney JT, Heredia O, Sane S, Lewis SC, Chang IK. A comparison of three methods for defining acute myocardical infarction. Academic Emergency Medicine 10(5):534. 2003.

11. Davis GK, Nimrod M. Cardiac biomarker usage in the West Indies. West Indian Medical Journal. 2003; 52:260-261.

12. de Lemos JA, Morrow DA. Combining natriuretic peptides and necrosis markers in the assessment of acute coronary syndromes. Reviews in Cardiovascular Medicine. 2003; 4:Suppl-46.

13. Lewandrowski K, Chen A, Januzzi J. Cardiac markers for myocardial infarction. A brief review. American Journal of Clinical Pathology. 2002; 118:Suppl-9.

14. Gensini GF, Fusi C, Conti AA, Calamai GC, Montesi GF, Galanti G, Noferi D, Carbonetto F, Palmarini MF, Abbate R, Vaccari M. Cardiac troponin I and Q-wave perioperative myocardial infarction after coronary artery bypass surgery. Critical Care Medicine. 1998; 26:1986-1990.

15. Barron JT. Cardiac troponin I and non-Q-wave myocardial infarction: how useful is it after coronary artery bypass surgery? Critical Care Medicine. 1998; 26:1936-1937. 
Norzeihan Jan Bappu graduated from the University of Edinburgh and is currently a surgical trainee in Department of Vascular Surgery, the New Royal Infirmary of Edinburgh. Her main interest is Cardiothoracic Surgery, and is actively involved in research on 'Blood Conservation in Cardiac Surgery'. Past publications include factors predicting loss and gain of red cell volume and perioperative blood volume change in cardiac surgery. She was awarded a Wellcome Trust Elective Award in 2005 for training on adult and paediatric surgery in AIIMS, India as well as undertaking biomedical research on troponin in Cardiac Surgery.

Professor P Venugopal is the Head of Department in Cardiovascular Surgery, and the Director of All India Institute of Medical Sciences, New Delhi, India. He has published over 150 articles on adult and paediatric cardiac surgery, and is one of the pioneers in cardiac stem cell research in India.

Mr Pankaj S Mankad is Consultant Cardiac Surgeon in the New Royal Infirmary of Edinburgh and was a former UK leading paediatric surgeon. He is also the Foundation Programme Director for postgraduate doctors, and is actively involved in various academic events in the Royal College of Surgeons, Edinburgh. His main interest is academic cardiac surgery.

16. Fellahi JL, Gue X, Richomme X, Monier E, Guillou L, Riou B. Short- and long-term prognostic value of postoperative cardiac troponin I concentration in patients undergoing coronary artery bypass grafting. Anesthesiology. 2003; 99:270-274.

17. Ascione R, Caputo M, Angelini GD. Off-pump Coronary Aretry Bypass Grafting: Not a Flash in the Pan. Annals of Thoracic Surgery. 2003; 75:306-13.

18. Angelini GD et al. Early and midterm outcome after off-pump and on-pump surgery in BEating Heart Against Cardioplegic Arrest Studies (BHACAS 1 and 2): a pooled analysis of two randomised controlled trials. Lancet. 2002; 359:1194.

19. Czerny $\mathrm{M}$ et al. Inflammatory response and myocardial injury following coronary artery bypass grafting with or without cardiopulmonary bypass. European Journal of Cardiothoracic Surgery. 2000; 17:737-742.

20. Ascione $\mathrm{R}$ et al. Inflammatory Response After coronary revascularisation with or without cardiopulmonary bypass. Annals of Thoracic Surgery. 2000; 69:1198-204. 